\title{
Molecularly Imprinted Polymers (MIP) Based Electrochemical Sensors for Detection of Endosulfan Pesticide
}

\author{
Yohandri Bow ${ }^{\#}$, Edy Sutriyono*, Subriyer Nasir $\$$, Iskhaq Iskandar ${ }^{\&}$ \\ ${ }^{\#}$ Chemical Engineering Department, State Polytechnic of Sriwijaya, Palembang, 30139, Indonesia \\ \& Environmental Science, Sriwijaya University, Indonesia \\ E-mail: yohandribow@gmail.com
}

*Geology Study Program, Sriwijaya University, Palembang, 30139, Indonesia

${ }^{\$}$ Chemical Engineering Department, Sriwijaya University, Palembang, 30139, Indonesia

${ }^{\circledR}$ Physics Department, Mathematics and Natural Science Faculty, Sriwijaya University, Palembang, 30139, Indonesia

\begin{abstract}
The use of endosulfan pesticides in agriculture can cause environmental problems, such as pollution in aquatic environments that can lead to the destruction of fishery resources and drinking water. So, it has become imperative to detect and separate the hazardous pesticide endosulfan from contaminated water. In this work, molecularly imprinted membrane has been fabricated for the specific recognition by using methacrylic acid (MAA) as functional monomer and ethylene glycol dimethyl acrylate (EGDMA) as cross-linker. Scanning Electron Microscopy (SEM) confirmed the molecular imprinting of endosulfan on membrane matrix. Sensing of the endosulfan by voltammetry followed this. The electrochemical potential is additional information enhancing the selectivity of the sensor. It can be concluded that MIP-based voltammetric sensors are very promising analytical tool for the development of highly selective analytical sensor. The test results of electrode performance indicated that MIP endosulfanbased aluminum-carbon sensor had a detection limit of $0.02 \mathrm{mM}$, sensitive in the concentration range from 0.02 to 0.12 $\mathrm{mM}$ with Nernst factor $>\mathbf{0 . 0 5 9} \mathrm{V} /$ decade and had good stability.
\end{abstract}

Keywords - endosulfan pesticide; molecularly imprinted polymer; electrochemical sensor

\section{INTRODUCTION}

Environmental contamination with pesticides is an undesired consequence of agricultural activities. Direct discharge of pesticide-containing wastewater into soil or natural water sources constitutes a major problem of pointsource contamination. Inappropriate handling of pesticides during agriculture use results in a risk of contamination in tasks such as the spraying of pesticides on the field, their dilution and pouring in spraying tanks, the discharge of residues and cleaning of those tanks after application, and utilization in postharvest treatment [1], [2].

In recent years, farmers are using a higher percentage of chemical fertilizers and pesticides to enhance the yield of crops to fulfil growing demands of food crops. If pesticides are not used, crop yield has been reported to reduce by about $10 \%$. It is well known that several pesticides used for agriculture purposes are associated with surface and groundwater contamination. Extravagant and enormous usage of endosulfan pesticide not only will leave a traceful damage for the environment but also to the human living in it. This condition leads to the concern for people living in certain_places such as children, pregnant women, farmers, farm workers and the elderly undergo negative health effects and can also cause acute poisoning, cancer, neurological damage, birth defects and reproductive as well as development harms. The excessive and uncontrolled uses of endosulfan pesticide in crops have raised and alarm for its toxicity not only in crops but also in drinking water. So, it has become imperative to detect and separate the hazardous pesticide endosulfan from contaminated water [3].

Persistent Organic Pollutants (POPs) are a set of chemicals that are toxic; persist in the environment for long periods of time, and biomagnify as they move up through the food chain. POPs have been linked to adverse effects on human health and animals. Because they circulate globally via the atmosphere, oceans, and other pathways, POPs released in one part of the world can travel to regions far from their source of origin [4]. 
Endosulfan is a man-made insecticide. It is used for control of a number of insects on such food crops as grains, tea, fruits, and vegetables and on such non-food crops like tobacco and cotton. It is also used as a wood preservative.

Endosulfan is sold as a mixture of two different forms of the same chemical (referred to as alpha- and betaendosulfan). It is a cream-to-brown colour solid that may appear crystalline or be in flakes. It has a distinct odour similar to turpentine. Endosulfan does not burn. Its chemical name is 1,4,5,6,7,7-hexachloro-8,9,10-trinoborn-5-en-2,3ylenebismethylene sulfite or $6,7,8,9,10,10$-hexachloro1,5,5a,6,9,9a-hexahydro-6,9-methano-2,4,3-benzodioxathiepen 3-oxide. The empirical, structural formula and molecular mass are given as Fig. 1 below.

$\mathrm{C} 9 \mathrm{H} 6 \mathrm{Cl} 6 \mathrm{O} 3 \mathrm{~S}$

406,9

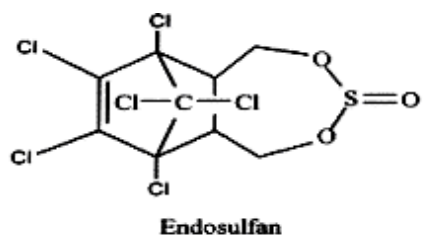

Fig. 1 Structure formula endosulfan

Endosulfan is an organochlorine insecticide and acaricide, and act as a contact poison in a wide variety of insects and mites [5]. It is easily absorbed by the stomach, lungs and through the skin, which meaning that all routes of exposure can pose a hazard. It enhances the effect of estrogens can pose a hazard. It enhances the effect of estrogens and acts as an endocrine disruptor, causing reproductive and development damage in animals and humans as well as cause cancer [6].

Endosulfan is a neurotoxic organochlorine insecticide of the cyclodiene family of pesticides. It is highly toxic and an endocrine disruptor and it is banned in the European Union, Philippine, and several countries. Due to extreme toxic of bioaccumulation and pollution in the environment, a worldwide ban for usage and production of endosulfan is applied as regulated by the Stockholm Convention [7].

Endosulfan is a broad-spectrum organochlorine insecticide and comes in $\alpha$ and $\beta$ isomers, both of which are found in commercial formulations. It is practically insoluble in water but is soluble in organic solvents. In the biological tissues and the environment, it is known to degrade into various metabolites, which include endosulfan sulphate, endosulfan alcohol, endosulfan ether and endosulfan lactone [8]. Consequently, it becomes the foremost important act to get the early detection and separation of this pesticide from the polluted water. Recent researches are reported developing methods in sensing and separating the pesticide from the environment. Biosensors and sensors are excellent tools for detecting the pesticides. Molecularly Imprinted Polymers (MIPs) are used for separating pesticides, which have created receptor structure artificially. There are many recent reports of molecularly imprinted polymer, which are used to develop detection systems for pharmaceuticals and environmental contaminants.

Molecular imprinting is a technology that is used to create specific cavities in the synthetic polymer with a memory for the molecules template. Until now molecularly imprinted polymers (MIPs) have earned a strong position in material science and technology, expanding the list of functional materials significantly.

Molecular imprinting technology is a promising technique for creating recognition elements for selected compounds and has been successfully applied for the synthesis of environmental pollutants such as pesticides [9].

The molecular imprinting technique continues to be a fascinating field of analytical chemistry offering strategies for creating molecule-specific recognition matrices with recognition capabilities analogous to those of biological receptors [10]. The positions, shape, and size of the functional groups in the sites of recognition generated are complementary to its original analyte. Thus, molecularly imprinted polymers (MIPs) rebind their original analyte in preference to related molecules. MIPs have considerable potential for application in the areas of clinical analysis, medical diagnostic, environmental monitoring and drug delivery. Imprinted polymer materials possess several other virtues viz. physical and chemical stability, storage endurance and imprint memory, which is essential for the preparation of recognition membranes in a robust and reusable sensing device. Moreover, MIPs are usually cheaper and more accessible high-affinity recognition materials in contrast to many biological entities [11].

MIPs have been synthesized for large and small molecules, herbicides including atrazine [12] and 2,4,5 trichlorophenoxyacetic acid [13], environmental contaminants pentachlorophenol and organophosphorus such as paraoxon, dichlorovos [14]. Scheme for molecular imprinting is given as Fig. 2 [15].

MIPs are advantageous for sensors because they selectively bind the targeted compound while ignoring all others. Therefore, an MIP-based sensor should reduce the number of false positives that often plague other types of sensors (for example, pesticides often cause false alarms with nerve gas sensors) [16].

Potentiometric sensors, a subgroup of chemical sensors, are attractive for practical applications, as they are associated with small size, portability and low energy consumption and cost compared to another group of sensors [17], [18].

The development of MIP-based sensors with potentiometric transduction does not require the template or print molecule to be extracted from the membrane to create membrane potential and does not have to diffuse through the membrane so that there is no size restriction on the template molecule, the main Achilles heel of MIP's until recently. MIP-based potentiometric sensor for methylphosphonic acid, an ultimate degradation product of nerve agents by coupling surface imprinting technique with a nanoscale transduces indium tin oxide [19]. The literature reveals that the prepared imprinted polymer membranes can be effectively used for the detection of nerve agents by fabricating them into potentiometric sensors [20], [21].

The purpose of this study is to develop a molecularly imprinted polymer composite membrane to identify and recognize the contaminant (endosulfan) selectively in the water. The functions of selective matrix membrane are the identification and remove of selected analyte. In this research, MIPs composite membrane has been synthesized 


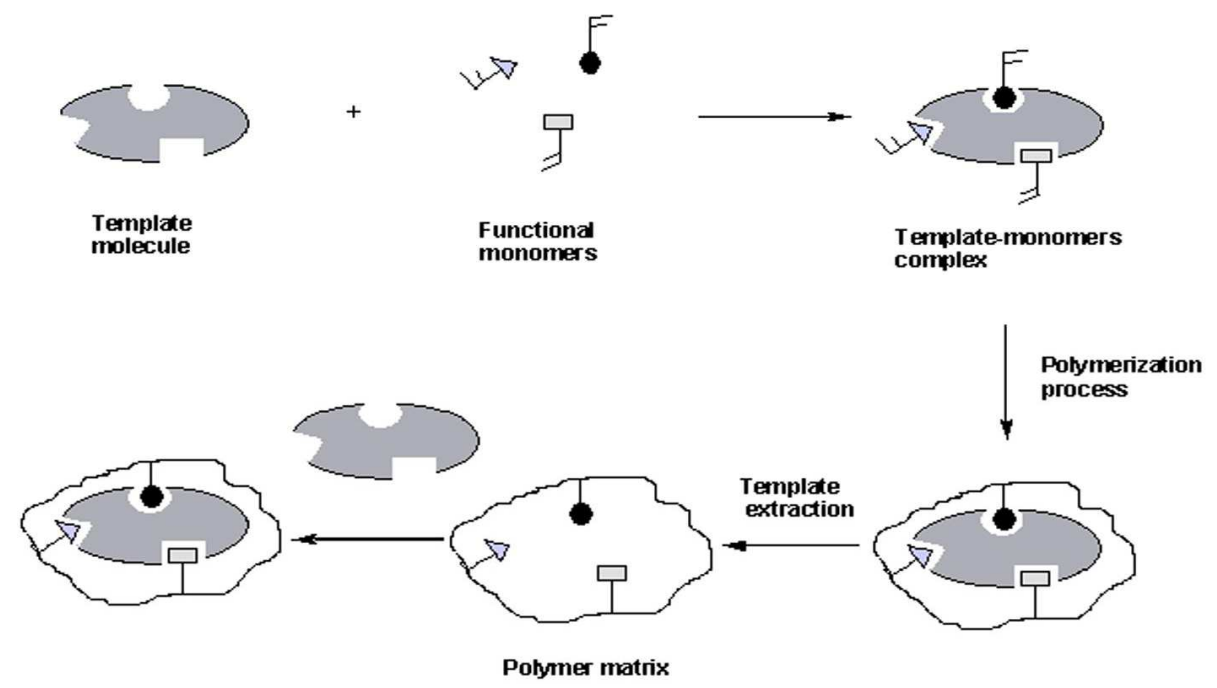

Fig. 2 Scheme of molecular imprinting

and used for sensing and separating the pesticide because of its exclusive stability and easy to adapt using an advanced instrumental technique like UV-Visible spectrophotometer and Scanning Electron Microscopy (SEM).

\section{MATERIAL AND METHOD}

\section{A. Preparation of Endosulfan Template}

All the reagents were of analytical grade. The procedure for the synthesis of MIP and NIP polymers as following procedure: Briefly, a template of endosulfan was taken in 0.025 gr endosulfan and $2.1 \mathrm{ml}$ chloroform solvent, $0.525 \mathrm{ml}$ EDMA cross-linker, $0.059 \mathrm{ml}$ MAA functional monomer and 0.05 gr BPO initiator were added to it. The entire components were thoroughly mixed for 15 minutes. Blank polymer without endosulfan was also prepared. After that, the reaction vial was kept at $-5^{\circ} \mathrm{C}$ in the freezer for 1 hour and kept at $70-120^{\circ} \mathrm{C}$ for 150 minutes for complete polymerization.

The polymer was transferred from the vial to mortal and ground. The polymers were collected through acetonitrile solvent filtered and washed using methanol and water (aquabidest) for 1 hour. Remaining residues filtrate was also collected for drying and future use.

A control sensor was prepared in every case following the same procedure but in the absence of template molecule. The control (or non-imprinted polymer-modified electrode) had the same treatment as the imprinted sensor, to ensure that the effect is observed only due to the imprinting features and not to the subsequent treatments undergone by the sensor.

\section{B. Removal of Template and Confirmation by UV-Visible Spectrophotometer}

Specific binding is confirmed by UV-Visible spectrophotometer. The MIPs were washed three times with methanol and also water to remove the print molecules. The imprinted template was eluted with methanol. The removal of the template was also confirmed by using a UV-Visible spectrophotometer.

\section{Characterization of MIP Composite Membrane by Scanning Electron Microscopy (SEM)}

Prepared molecular imprinted composite membranes with and without template were categorized by using scanning electron microscopy (SEM, Model: Pro-X) and the micrograph was recorded of all the different samples of the membrane including non-imprinted and MIP composite membranes.

\section{Electrochemical Sensor Preparation}

Electrochemical studies were performed using a potentiostat. The general procedure used to prepare the potentiometric sensor based MIP, using aluminium wire (1 $\mathrm{mm}$ diameter and $120 \mathrm{~mm}$ long) and carbon. The electrodes were polished with fine alumina slurries with a polishing cloth, sonicated in distilled water and dried in air. An unsaturated resin was glued to one end of the above aluminium wire. Endosulfan was dipped into the aluminium wire and added a solution of chloroform on the sensor surface that has been coated with endosulfan.

\section{RESULTS AND DISCUSSION}

\section{A. MIP Endosulfan Characterization}

Scanning Electron Microscopy (SEM) study usually used for recognizing the morphology structure and MIP surface because it has an excellent and very high resolution. The morphology surface SEM images of the IP endosulfan template under the magnificent 500X - 1.99KX and 30 resolution are shown in Fig. 3-6. SEM micrograph of MIP at various magnifications which generally shows rough MIP surface with the irregular pores. The globular and porous MIP has higher adsorption capacity toward the target (in this case endosulfan) compared to a NIP, possibly due to the porosity and higher surface area. The specific binding sites created also contribute to the higher adsorption of MIP towards endosulfan.

SEM analysis described both the morphological characteristics of the shape and surface structure of the MIP. 
This was important information for the synthesis and applications of MIP.

Fig. 3, 4, 5 and 6 show the SEM micrographs of the MIP composite membrane imprinted by endosulfan template, which was recorded at 500X, $1 \mathrm{KX}, 1.5 \mathrm{KX}$ and $1.99 \mathrm{KX}$ magnifications and at a resolution of $30 \mu \mathrm{m}$. The surface texture clearly exhibits the cavities on the surfaces and within the pores, which is a clear indication of a change of porous behavior of MIP composite membrane after removal of the template. These things change in porous characteristic behavior in thermodynamical studies. Here were the results of SEM to the MIP obtained [22].

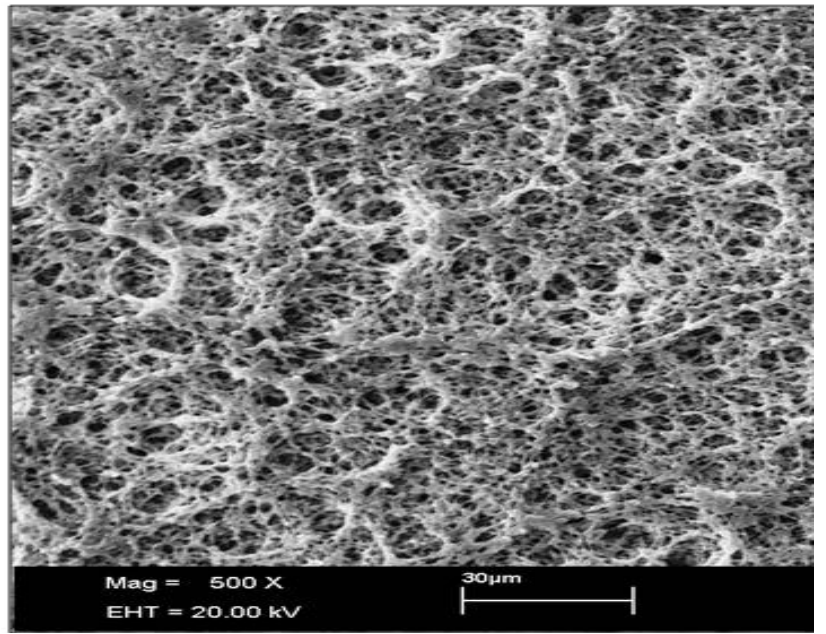

Fig. 3 SEM of the MIP endosulfan template at 500X magnification and 30 $\mu \mathrm{m}$ resolution

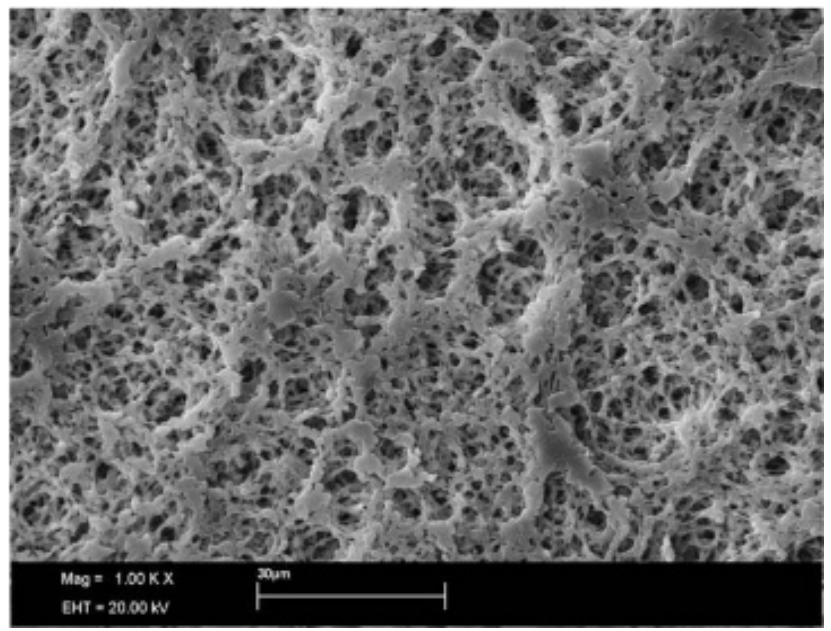

Fig. 4 SEM of the MIP endosulfan template at 1.0 KX magnification and $30 \mu \mathrm{m}$ resolution

\section{B. Specific Binding of Endosulfan}

MIP has cavities that specific, which can be passed by template because every template has its sensitivity. Endosulfan MIP sensitivity can be measured using the UVVisible Spectrophotometer and see the elution curve. From the curve, it can be known that MIP has a sensitivity to the template. Fig. 7 shows the graph of absorbance vs. wavelength of endosulfan concentration passed from MIP and non-imprinted polymer.

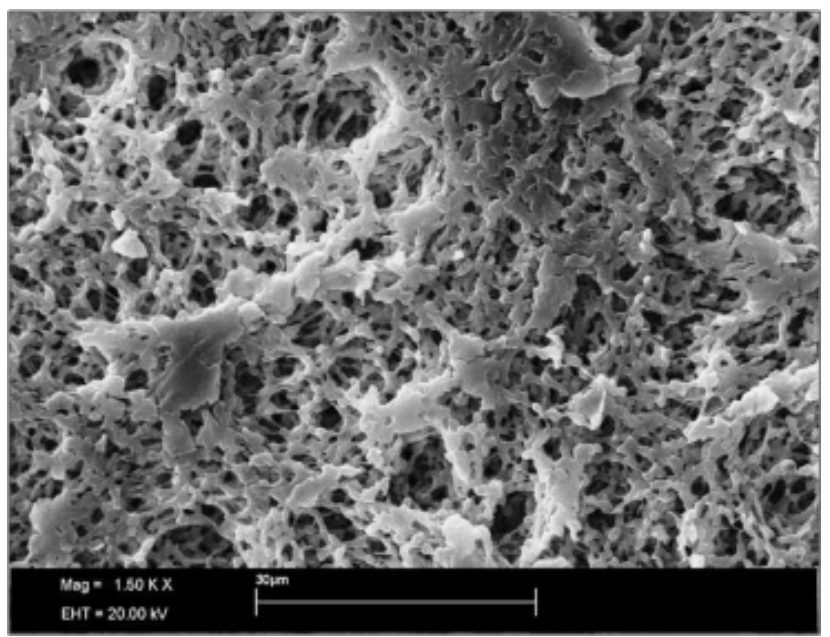

Fig. 5 SEM of the MIP endosulfan template at $1.5 \mathrm{KX}$ magnification and 30 $\mu \mathrm{m}$ resolution

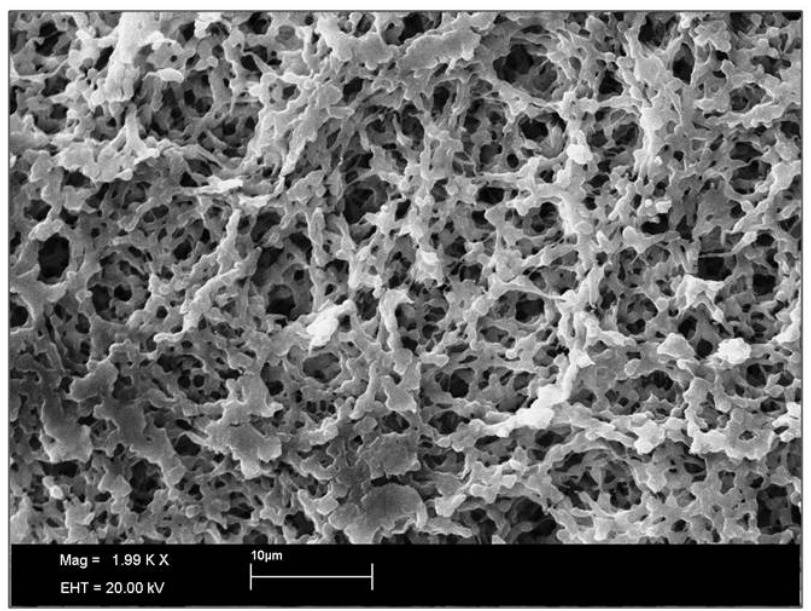

Fig. 6 SEM of the MIP endosulfan template at $1.99 \mathrm{KX}$ magnification and $30 \mu \mathrm{m}$ resolution

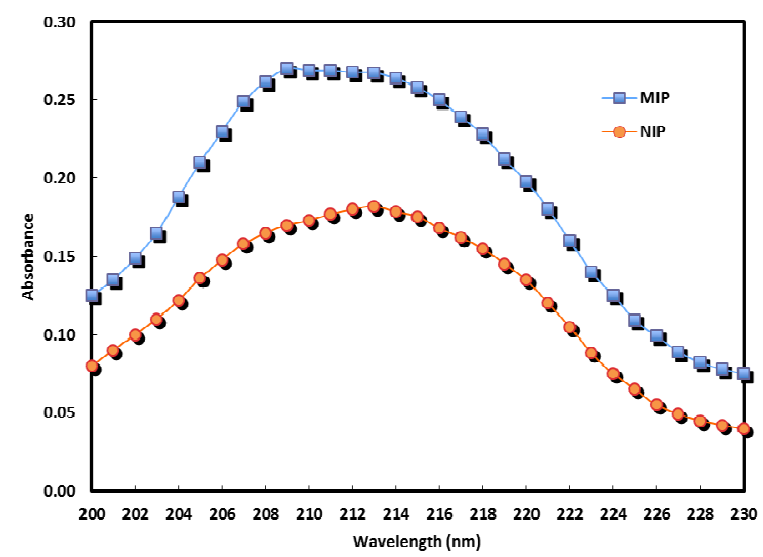

Fig. 7 Endosulfan concentration passed from MIP and NIP

\section{The Concentration of Endosulfan Bound to the MIP}

MIP endosulfan was fabricated with cooling - heating method for a variable heating time (90, 120, and 150 minutes), which includes the initial concentration (target), endosulfan concentrations of free and bound endosulfan concentrations of MIP. The relationship between the concentrations of endosulfan independent of the 
concentration of endosulfan bound to the MIP can be seen in Fig. 8.

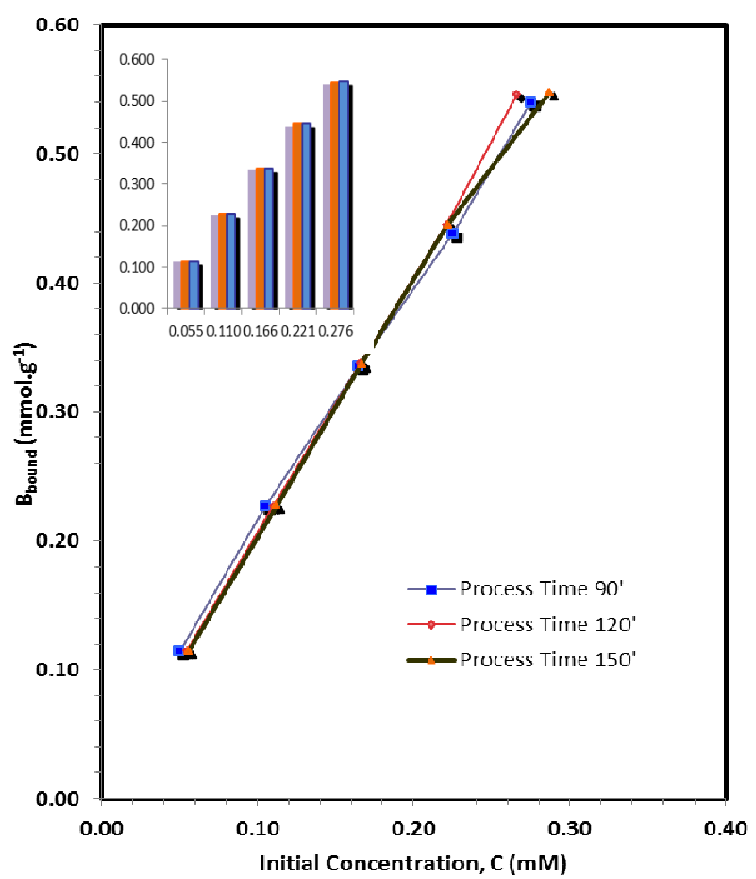

Fig. 8 The relationship between the concentrations of target bounds to the initial concentration of MIP

Furthermore, the concentration of endosulfan independent of the concentration of bound target can be seen in Fig. 8. It was explained that the polymer bound endosulfan concentrations increased with increasing concentrations of endosulfan free. Heating time of 150 minutes produces the least amount of free endosulfan than the heating time of 90 minutes and 120 minutes. This indicates that endosulfan bounds to the MIP are most numerous in the old polymer prepared by heating 150 minutes. This result corresponds to the available cavities, where these polymers are more porous than the other polymers.

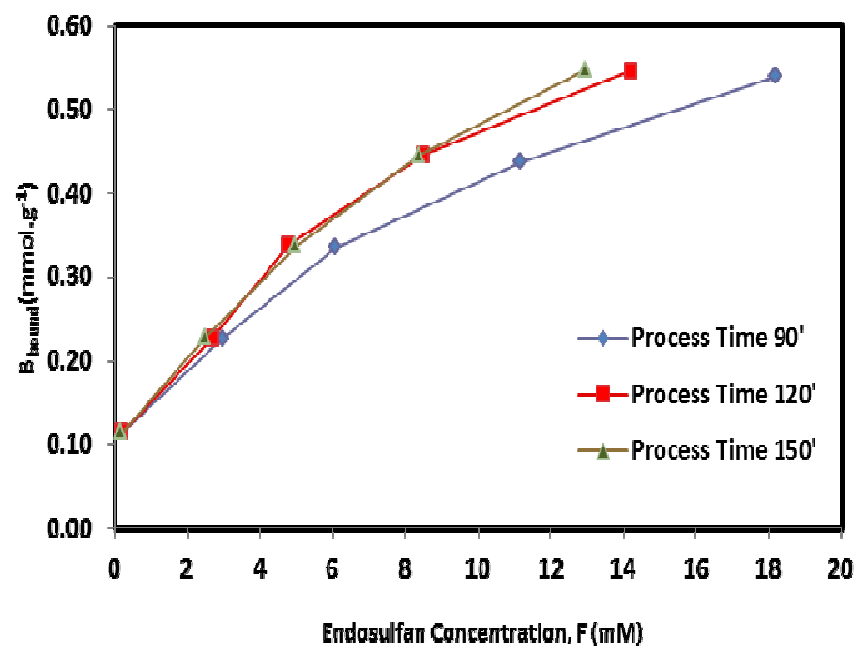

Fig. 9 The relationship between $\mathrm{B}_{\text {bound }}$ to the concentration of endosulfan free

From Scatchard Plots, it was found that the maximum amount of targets bounds to the MIPs was $10,03 \mathrm{mmol} / \mathrm{gr}$ for heating time 150 minutes, respectively. On the other hand, the cavities of MIPs were 528 for heating time 150 minutes, as analyzed from the SEM images. These finding confirmed that the heating time of 150 minutes results in the endosulfan MIP with the best endosulfan sensing properties. The relationship between the concentrations of bounds to the initial concentration can be seen in Fig. 10.

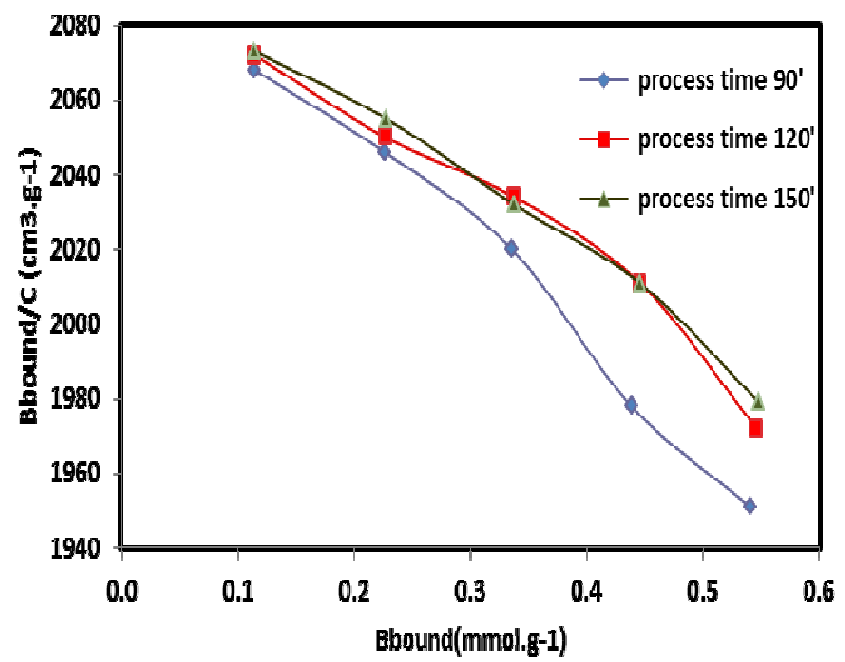

Fig. 10 Relationship $\mathrm{B}_{\text {bound }} / \mathrm{C}$ to $\mathrm{B}_{\text {bound }}$

\section{Sensor Performance Test}

After testing the performance of the sensor by measuring the voltage (volts) on a standard solution with varying concentrations, linearity equation and Nernst factor were obtained.

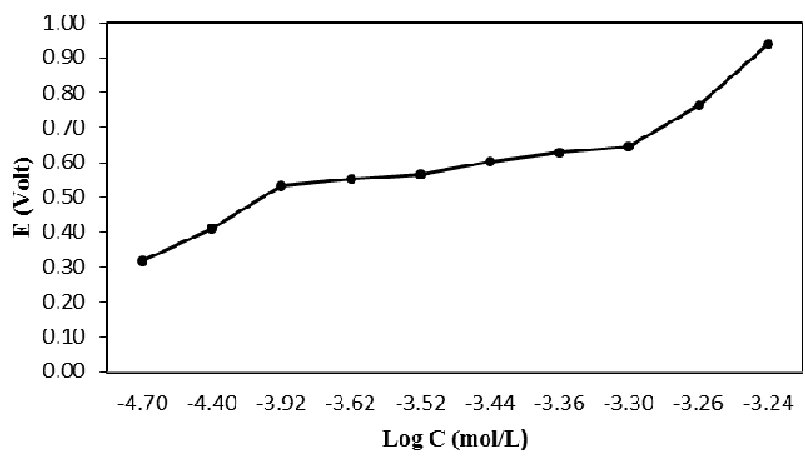

Fig. 11 Graph between MIP endosulfan potential to the target logarithm concentration on the aluminum-carbon sensor on the $1^{\text {st }}$ day

Fig. 11 showed that the slope of the function E versus log concentration increased with increasing concentrations of the target value. Based on the graph, linear equation with three ranges of concentration was obtained. Based on the results of a linear regression on the graph of potential E (volts) to the logarithm concentration, the obtained parameter values were as in Table 1.

From the measurements performed in the concentration range from 0.02 to $0.58 \mathrm{mM}$ obtained three $\mathrm{N}$ slopes with a value of 0.2682 in the concentration range from 0.02 to 0.12 $\mathrm{mM}, 0.3093$ in the concentration range from 0.24 to 0.44 $\mathrm{mM}$, and 4.2808 in the concentration range from 0.50 to 0.58 $\mathrm{mM}$. These sensors had good sensitivity in the concentration range from 0.02 to $0.12 \mathrm{mM}$ as indicated by the linearity value closed to $1, \mathrm{R}^{2}=0.9977$. 
TABLE I

The ObTained Parameters of Potential Plot E (Volt) to THE LOGARITHM CONCENTRATION AT 1ST DAY

\begin{tabular}{|c|c|c|c|}
\hline $\begin{array}{c}\text { Concentrati } \\
\text { on Range }\end{array}$ & $\begin{array}{c}\mathbf{E}=\mathbf{K}+\mathbf{N} \text { (slope) Log } \\
\mathbf{C}\end{array}$ & $\mathbf{Z}$ & $\mathbf{R}^{\mathbf{2}}$ \\
\hline $0.02-0.12$ & $1.5838+0,2682 \log \mathrm{C}$ & 0.21626 & 0.9977 \\
\hline $0.24-0.44$ & $1.6656+0,3093 \log \mathrm{C}$ & 0.18752 & 0.9841 \\
\hline $0.50-0.58$ & $14.763+4,2808 \log \mathrm{C}$ & 0.01355 & 0.9120 \\
\hline
\end{tabular}

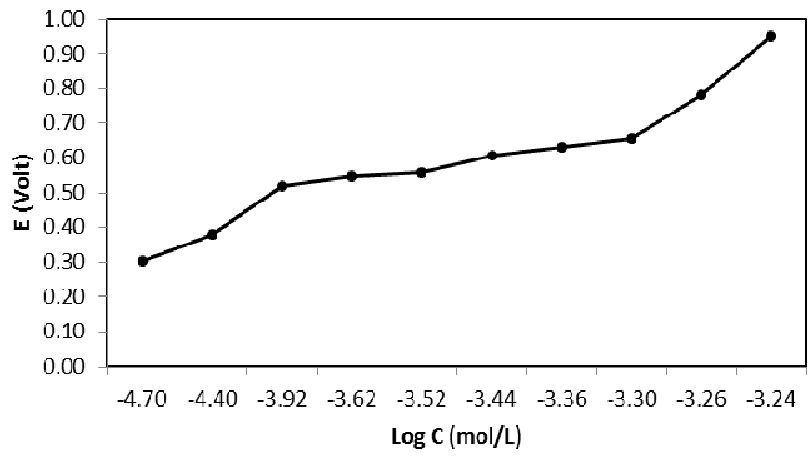

Fig. 12 Graph of MIP potential of endosulfan to the target of logarithm concentration on the aluminum-carbon electrodes on at $20^{\text {th }}$ day

Based on the results of a linear regression on a graph of potential $\mathrm{E}$ (volt) to the logarithm of the concentration, the obtained parameters were as Table 2 .

TABEL II

OBTAINED PARAMETERS FROM E (VOLT) POTENTIAL Plot TO THE LOGARITHM OF THE CONCENTRATION AT THE 20TH DAY

\begin{tabular}{|c|c|c|c|}
\hline $\begin{array}{c}\text { Concentration } \\
\text { Range }\end{array}$ & $\mathbf{E}=\mathbf{K}+\mathbf{N}$ (slope) Log & $\mathbf{Z}$ & $\mathbf{R}^{\mathbf{2}}$ \\
\hline $0,02-0,12$ & $1,631+0,2837 \log \mathrm{C}$ & 0,20444 & 0,9993 \\
\hline $0,24-0,44$ & $1,7386+0,3302 \log \mathrm{C}$ & 0,17565 & 0,9213 \\
\hline $0,5-0,58$ & $14,87+4,3094 \log \mathrm{C}$ & 0,01346 & 0,9330 \\
\hline
\end{tabular}

From the measurements done on the $20^{\text {th }}$ day in the concentration range from 0.02 to $0.58 \mathrm{mM} \mathrm{N}$ obtained three slopes with a value of 0.2837 in the concentration range from 0.02 to $0.12 \mathrm{mM}, 0.3302$ in the concentration range of $0.24-0.44 \mathrm{mM}$, and 0.3094 in the concentration range 0.50 to $0.58 \mathrm{mM}$ Measurement on the $20^{\text {th }}$ day also showed sensors had good sensitivity in the concentration range from 0.02 to $0.12 \mathrm{mM}$ with the linearity of 0.9993 .

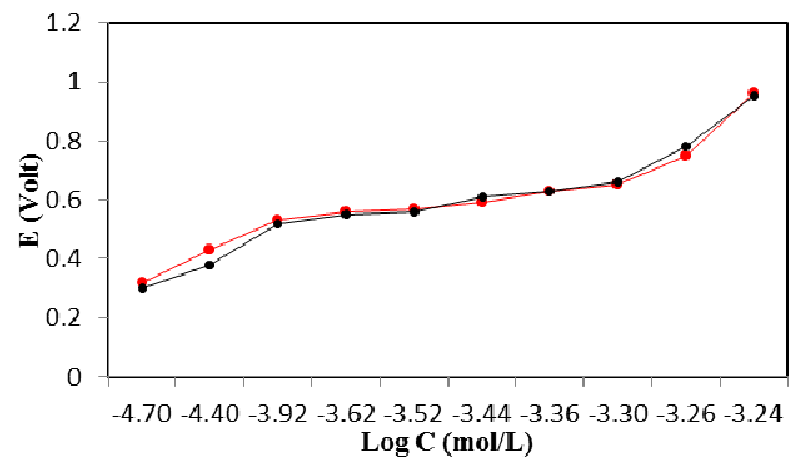

Fig. 13 Graph of stability of sensor the $1^{\text {st }}$ and the $20^{\text {th }}$ days
Based on Fig. 13, measurements of the $1^{\text {st }}$ day and $4^{\text {th }}$ were coincided, this indicated that this sensor had good stability.

The limit of detection indicated the lowest concentration of analyte ions addressed by the sensors the lowest measurement limit. Based on the experiment, the detection limit of the aluminum-carbon sensor was $0.02 \mathrm{mM}$ Sensors responded well to a minimum level of $0.02 \mathrm{mM}$ concentration of endosulfan.

\section{CONCLUSIONS}

Molecularly imprinted polymers were successfully prepared for endosulfan using MAA as functional and EGDMA as cross-linking monomers. The method developed for the determination of residues of endosulfan. The molecularly imprinted polymer matrices specific to endosulfan pesticides were synthesized and used for the fabrication of MIP composite membranes. These MIP composite membranes were further characterized by using UV-Visible and SEM. In recent studies, for the specific recognition of endosulfan pesticide, the molecularly imprinted membrane is fabricated, widely used in controlling insects.

The test results of electrode performance indicated that MIP endosulfan-based aluminum-carbon sensor had a detection limit of $0.02 \mathrm{mM}$, sensitive in the concentration range from 0.02 to $0.12 \mathrm{mM}$ with Nernst factor $>0.059 \mathrm{~V} /$ decade and had good stability.

\section{REFERENCES}

[1] Carlos, et al. On-farm Biopurification Systems: Role of White Rot Fungi in Depuration of Pesticide-containing Wastewater. Minireview. FEMS Microbial Lett. 345. p. 1-12. 2013.

[2] Singh, et al. Selective Recognition of Endosulfan Pesticide in Environmental Matrix with Molecularly Imprinted Polymer Membrane. Research Journal of Chemical Science, Vol. 4(4), p. 6370, 2014.

[3] Juan He, Lixin Song, Si Chen, Yuanyuan Li, Hongliang Wei, Dongxin Zhao, Keren Gu and Shunsheng Zhang. Novel Restricted Access Materials Combined to Molecularly Imprinted Polymers for Selective Solid-Phase Extraction of Organophosporous Pesticides from Honey. Food Chemistry, Elsevier. 187. p. 331-337. 2015.

[4] Kanchan Singh, Akmal Pasha, B.E. Amitha Rani. Preparation of Molecularly Imprinted Polymers for Heptachlor: An Organochlorine Pesticide. Chronicles of Young Scientists, Vol. 4(1). p. 46-50. 2013. doi: 10.4103/2229-5186.108806.

[5] Abdul Latif Ahmad, Nuur Fahanis Che Lah and Siew Chun Low. Molecular Imprinted Polymer for Atrazine Detection Sensor: Preliminary Study. Chemical Engineering Transactions. Vol. 45. p. 1483-1488. 2015. doi: 10.3303/CET1545248.

[6] Maricely Janette Uria Toro, Luiz Diego Marestoni and Maria Del Pilar Taboada Sotomayor. A New Biomimetic Sensor Based on Molecularly Imprinted Polymers for Highly Sensitive and Selective Determination of Hexazinone Herbicide. Sensors and Actuators B 208. p. 299-306. 2015

[7] Jianshe Tang and $\mathrm{Li}$ Xiang. Development of a Probe Based on Quantum Dots Embedded with Molecularly Imprinted Polymers to Detect Parathion. Pol. J. Environ. Stud. Vol. 25, No. 2. p. 787-793. 2016. doi: $10.15244 /$ pjoes/60888.

[8] Fabrizio Ruggieri, Luca Lozzi, Angelo Antonio and Sandro Santucci Development of Molecularly Imprinted Polymeric Nanofibers by Electrospinning and Applications to Pesticide Adsorption: Sample Preparation. Journal Separation Science. February 2015. doi: 10.1002/jssc.201500033.

[9] Singh, et al. Molecular Imprinting for Heptachlor. Cronicles of Young Scientists. Vol. 4, Issue 1 Jan-Jun. p. 46-50. 2013. 
[10] Vishnuvardhan, Varada, et al. Imprinted Polymer Inclusion Membrane Based Potentiometric for Determination and Quantification of Diethyl Chlorophosphate in Natural Waters. American Journal of Analytical Chemistry, 2, p. 376-382, 2011.

[11] Tehrani, M.S, et al. Molecularly Imprinted Polymer Based PVCMembrane-Coated Graphite Electrode for Determination of Metoprolol. Int. J. Electrochem. Sci. 5. p. 88-104. 2010.

[12] Saliza Asman, Sharifah Mohamad and Norazilawati Muhamad Sarih. Exploiting B-Cyclodextrin in Molecular Imprinting for Achieving Recognition of Benzylparaben in Aqueous Media. Int. J. Mol. Sci. 16. p. 3656-3676. 2015. doi: 10.3390/ijms16023656.

[13] Gabycarmen Navarrete-Rodriguez, Cesareo Landeros-Sanches and Alejandra Soto-Estrada. Endosulfan: Its Isomers and Metabolites in Commercially Aquatic Organisms from the Gulf of Mexico and the Caribbean. Journal of Agriculture Science, Vol. 8(1). p. 8-24. 2016. doi: $10.5539 /$ jas.v8n $1 \mathrm{p} 8$.

[14] U.S. Environmental Protection Agency, Manual of Analytical Methods for the Analysis of Pesticides in Human and Environmental Samples. 2012

[15] Sha Yang, Yonghui Wang, Yingda Jiang, Shuang Li and Wei Liu. Molecularly Imprinted Polymers for the Identification and Separation of Chiral Drugs and Biomolecules. Polymer. 8. 2016. doi: 10.3390/polym8060216.

[16] Saxena, Ronika and Poonam Garg. Vitamin E Provides Protection against in vitro Oxidative Stress due to Pesticide (Chlorpyrifos and Endosulfan) in goat RBC. GERF Bulletin of Biosciences 1(1), p. 1-6, 2010

[17] Rusdianasari, Bow, Y., Taqwa, A. Treatment of coal stockpile wastewater by electrocoagulation using aluminum electrode. J. Advanced Material Research. Vo. 896. p. 145-148. 2014.
[18] Rusdianasari, Meidinariasty, and Purnamasari, I. Level Decreasing Kinetics Model of Heavy Metal Contents in the Coal Stockpile Wastewater with Electrocoagulation. International Journal on Advanved Science, Engineering and Information Technology. Vol. 5(6). p. 387-391. 2015. doi:http://dx.doi.org/10.18517/ijaseit.5.6.593.

[19] Mercedes Roldan-Pijuan, Rafael Lucena, Soledad Cardenas, Miguel Valcarcel, Abuzar Kabir and Kenneth G. Furton. Stir Fabric Phase Sorptive Extraction for the Determination of Triazine Herbicides in Environmental Waters by Liquid Chromatography. Journal of Chromatography A. 2014.

[20] Mengchun Zhou, Nana Hu, Shaohua Shu and Mo Wang. Molecularly Imprinted Nanomicrospheres as Matrix Solid-Phase Dispersan Combined with Gas Chromatography for Determination of Four Phosphorothioate Pesticides in Carrot and Yacon. J. Anal. Methods Chem. 2015. Doi: 10.1155/2015/385167.

[21] Monireh Khadem, Farnoush Faridbud, and Parviz Nouzi. Biomimetic Electrochemical Sensor Based on Molecularly Imprinted Polymer for Dichloran Pesticeide Determination in Biological and Environmental Samples. J. Iran Chem. Soc. Vol. 13(11). 2016. Doi 10.1007/s13738-016-0925-8.

[22] Singh K.P., Kumar Ajeet and Tyagi Shweta. Selective Recognition of Endosulfan Pesticide in Environmental Matrix with Molecularly Imprinted Polymer Membrane. Research Journal of Chemical Sciences. Vol.4(4). Pp. 63-70. 2014 